\title{
Performance analysis of different modulation schemes using OFDM techniques in Rayleigh fading channel
}

\author{
Mohammed Safiqul Islam, Gouri Rani Barai , Atiq Mahmood \\ Electrical and Electronic Department, American International University-Bangladesh (AIUB) \\ Safiqul_bd89@yahoo.com
}

(Received Feb 2011; Published March 2011)

\begin{abstract}
This paper deals with the communication system that uses M-ary Phase-Shift-Keying (M-PSK) and M-ary Quadrature Amplitude Modulation (M-QAM) to transmit information using OFDM technique over Rayleigh communication channel. In terms of Symbol Error Rate (SER), the performance of different modulation schemes using OFDM techniques in Rayleigh channel is analyzed. For different types of modulation schemes (QPSK, 16-QAM, 16-PSK, 64-QAM, 64-PSK) gray coded bit mapping has been used with OFDM techniques. So, better performance is shown in different modulation scheme by using gray coded bit mapping. Here in this paper, it is our goal to show the way through which the SNR result varies between M-PSK and M-QAM.
\end{abstract}

Keywords: OFDM, Gray Coded Bit Mapping, SER, Rayleigh fading, M- ary PSK, M- ary QAM

https://doi.org/10.14331/ijfps.2011.330006

\section{INTRODUCTION}

In recent years high speed wireless data communications have found many application areas like Orthogonal Frequency Division Multiplexing (OFDM) (R. D. Van Nee and R.Pras ).The principles of OFDM modulation have been used since the 1960s. But in recent years, this technology has crossed the limitations into the real world of modern communication systems to combat Inter Symbol Interference (ISI) through multicarrier modulation (John A.C Bingham, Mehul Jain and et al).

OFDM has proved to be very effective in mitigating adverse multipath effects of a broadband wireless channel. Counteracting the frequency selectivity of multipath channels by multiplexing information on different orthogonal carriers is the key to the OFDM success. Indeed, if a cyclic prefix is inserted between successive OFDM symbols, the overall system can be viewed as composed of $\mathrm{N}$ parallel frequency flat channels (Z.Wang and G. B. Giannakis and L. J. Cimini Jr).

So, now it becomes the underlying technology for various new applications such as digital audio broadcast (ETSI), digital video broadcasting-terrestrial (DVB-T) (ETS), wireless LAN (802.11a\&g, 802.16a\&b and Hiper LAN-2(ETSI)), broadband wireless (MMDS, LMDS), xDSL, and home networking. Actually, as of today, OFDM has replaced DSSS for 802.11a and $802.11 \mathrm{~g}$ wireless LAN application. In 802.11 and 802.16 , there are several data modulation schemes that are used with OFDM, such as Binary Phase Shift Keying (BPSK), quadrature amplitude modulation (QAM) to cover data rates for different needs. The bit error rate (BER) performance of these systems evaluated in additive white Gaussian noise (AWGN) channel (Nor K. Noordin et. al). In this technique, modulation, mapping rate are dynamically adapted based on channel condition to increase the system performance in terms of Bit Error Rate (BER) and throughput (bps) in various conditions such as channel mismatch, Doppler spreads, fading, etc.

By using gray bit mapping in different types of modulation schemes, bit constellation is rearranged where every adjacent bit constellation differ by only one bit. Symbol energy and symbol error rate also converted into bit energy and bit error rate to reduce bit error rate for different types of modulation schemes. In this paper, we are trying to figure out the way, which is Rayleigh fading channel for improving the performance of transmitting information.

We present the average SER performance for different M-PSK and M-QAM and use gray coded bit mapping for different modulation schemes to get BER performance with OFDM technique in Raleigh fading channel. The numerical results are computed and plotted for $\mathrm{M}=16$ and 64 .

The rest of the paper has been organized as follows: In section II, OFDM system and Rayleigh fading channel have been 
described. In section III, simulations and results are presented. Finally, we conclude the paper in the last section.

\section{System Model, (A. ChanNel Model)}

In a multipath environment, it is reasonably intuitive to visualize that an impulse transmitted from the transmitter will reach the receiver as a train of pulses. When there are large numbers of paths, applying Central Limit Theorem, each path can be modeled as circularly complex Gaussian random variable with time as the variable. This model is called Rayleigh fading channel model. A circularly symmetric complex Gaussian random variable is of the form,

$$
Z=X+\mathrm{jY}
$$

where real and imaginary parts are zero mean independent and identically distributed Gaussian random variables. For a circularly symmetric complex Gaussian random variable $Z$,

$$
E[z]=E\left[e^{j \theta} Z\right]=e^{j \theta} E[Z] .
$$

The statistics of a circularly symmetric complex Gaussian random variable is completely specified by the variance, $\sigma^{2}=$ $E\left[Z^{2}\right]$. Now, the magnitude $|Z|$ which has a probability density,

$$
p(z)=\frac{z}{\sigma^{3}} e^{\frac{-z^{2}}{2 \sigma^{2}}} z \geq 0
$$

is called a Rayleigh random variable. This model, called Rayleigh fading channel model, is reasonable for an environment where there are large number reflectors.

\section{B. OFDM transceiver:}

Before transmitting information bit in Raleigh fading channel through the OFDM transmitter we use different modulation schemes and also gray bit mapping, which is shown in Fig.1. And information data are modulated in a baseband fashion by the IFFT. Then the data is transmitted to the channel. The receiver performs the inverse process of the transmitter.

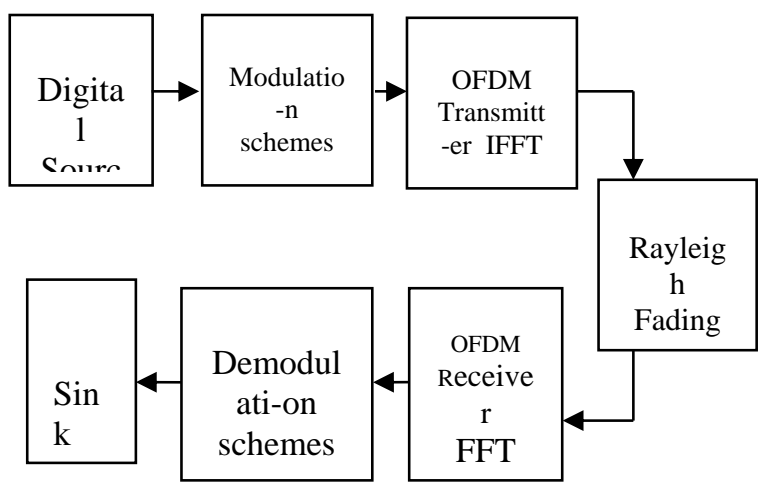

Fig. 1: Block diagram of OFDM transceiver in Ravleigh Fading channel.

In Orthogonal Frequency Division Multiplexing, multiple sinusoidal with frequency separation $\frac{1}{T}$ is used where $T$ is the symbol period. The sinusoids used OFDM can be defined as (John-R-Barry et al):

$$
g_{k}(t)=\frac{1}{\sqrt{T}} e^{\frac{j 2 \pi k t}{T}} w(t)
$$

Where $k=0,1, \ldots K-1$ correspond to the frequency of the sinusoidal and $w(t)=u(t)-u(t-T)$ is a regular window over $[0 \mathrm{~T}]$. OFDM uses multiple sinusoidals having frequency separation $\frac{1}{T}$ where each sinusoidal gets modulated by independent information. The information is multiplied by the corresponding carrier signals. Mathematically, the transmit signal is,

$$
\begin{gathered}
s(t)=a_{0} g_{0}(t)+a_{1} g_{1}(t)+\ldots+a_{K-1} g_{K-1}(t) \\
=\sum_{0}^{K-1} a_{k} g_{k}(t) \\
=\frac{1}{\sqrt{T}} \\
\sum_{0}^{K-1} a_{k} e^{\frac{j 2 \pi k t}{T}} w(t)
\end{gathered}
$$

The interpretation of the Eq. (5) is as follows:

(a) Each information signal $a_{k}$ multiplies the sinusoidal having frequency of $\frac{k}{T}$.

(b) Sum of all such modulated sinusoidal are added and the resultant signal is sent out as $s(t)$. The sampled version of the above equation is,

$$
s(n T)=\frac{1}{\sqrt{T}} \sum_{0}^{K-1} a_{k} e^{\frac{j 2 \pi k n t}{T}} w(n t)
$$

This operation corresponds to an inverse Discrete Fourier Transform (IDFT) operation. For different modulation selected parameters of the OFDM system based on IEEE 802.11a specifications are tabulated in Table-I.

Table-I: Selected Simulation Parameters

\begin{tabular}{l|c}
\multicolumn{1}{c|}{ Parameter } & Value \\
\hline Modulation & QPSK/16-QAM/64-QAM/16- \\
PFT size. nFFT & 64 \\
Number of used subcarriers. nDSC & 52 \\
FFT Sampling frequency & $20 \mathrm{MHz}$ \\
Subcarrier spacing & $312.5 \mathrm{kHz}$ \\
Used subcarrier index & $\{26$ to $1,+1$ to +26$\}$ \\
Cylcic prefix duration, Tcp & $0.8 \mathrm{us}$ \\
Data symbol duration, Td & $3.2 \mathrm{us}$ \\
Total Symbol duration, Ts & $4 \mathrm{us}$
\end{tabular}

From Table-I, we can see that the symbol duration $T=3.2 \mu \mathrm{s}$. This implies that the used subcarriers are $\frac{ \pm k}{T}=$ $\pm 312.5 \mathrm{kHz}, \pm 625 \mathrm{kHz} \ldots$. and so on. The available bandwidth of $20 \mathrm{MHz}$ is split into 64 subcarriers. Out of the 64 subcarriers 
having indices $k=[-32 \ldots .+31]$, the number of used subcarriers is 52. The used subcarriers having indices $[-26 . .-1+1 \ldots+26]$ are used for transmitting information sequence $a_{1}$ to $a_{52}$. Once the bits in each symbol are assigned to appropriate IDFT (Weinstein, S.B., Ebert, P.M) inputs or IFFT (inverse fast Fourier Transform) inputs, which is mathematically equivalent to IDFT operations, it transforms the frequency domain inputs into time domain signals. The length of the OFDM symbol is $N T$, where $T$ is the IFFT input symbol period mentioned above and $N$ is number of subcarriers of the system. After some additional processing, the time domain signal is transmitted across the channel. At the receiver, an FFT block is used to process the received signal and bring it into the frequency domain. When the output samples of the FFT are plotted in the complex plane, it forms a constellation, such as 16-QAM.

\section{Cyclic prefix}

In an OFDM transmission, we know that the transmission of cyclic prefix does not carry extra information. The signal energy is spread over time $T_{d}+T_{c p}$ whereas the bit energy is spread over the time $T_{d}$ i.e. $E_{s} .\left(T_{d}+T_{c p}\right)=E_{b} . T_{d}$. Simplifying,

$$
E_{s}=\frac{T d}{(T d+T c p)} E_{b}
$$

\section{Relation between $E_{b} / N_{o}$ and $E_{s} / N_{o}$ in OFDM}

The relation between symbol energy and the bit energy is as follows:

$$
\frac{E_{S}}{N_{0}}=\frac{E_{b}}{N_{0}}\left(\frac{n \cdot D S C}{n \cdot F F T}\right)\left(\frac{T d}{T d+T c p}\right)
$$

Expressing in decibels,

$$
\frac{E_{S}}{N_{0}} d B=\frac{E_{b}}{N_{0}} d B+10 \log _{10}\left(\frac{n \cdot D S C}{n \cdot F F T}\right)+10 \log _{10}\left(\frac{T d}{(T d+T c p)}\right)
$$

\section{E. M-ary PSK Modulation in OFDM Model}

The general analytic expression for M-ary PSK waveform is:

$s_{i}(t)=A \cdot \cos \left(w_{c} t+\emptyset_{i}(t)\right) ; i=0,1,2, . M-1$

Where $=\sqrt{\frac{2 E_{S}}{T_{S}}}, \emptyset=\frac{2 \cdot \pi \cdot m}{M}, m=0,1,2, M-1$

The parameter $E_{S}$ is symbol energy, $T_{S}$ is symbol time duration, and $0 \leq t \leq T_{s}$. For BPSK modulation, $M=2$, and for QPSK modulation $M=4$, and the modulation data signal shifts the phase of the waveform, $s_{i}(t)$.

The BPSK bandwidth efficiency is $1 \mathrm{bit} / \mathrm{Hz}$, while QPSK bandwidth efficiency is $2 \mathrm{bits} / \mathrm{Hz}$.

\section{SER calculation for M-ary PSK in Rayleigh fading channel}

The error rates of binary modulation schemes in a Rayleigh fading environment are available in closed form (John Proakis 1983). Closed-form expression for symbol error rate of M-ary Coherent Phase Shift Keying (CPSK) signals over Rayleigh fading channel can be expressed in terms of a single integral as Coherent PSK (Pawula, R.F et al):

$$
P_{e}(\gamma)=\frac{1}{\pi} \int_{-\frac{\pi}{2}}^{\frac{\pi}{2}-\alpha} \exp \left(-\gamma \sin ^{2} \alpha \sec ^{2} \theta\right) d \theta
$$

where $\gamma$ is the signal o noise power ratio, $\mathrm{M}$ is the number of modulation level and $\alpha=\frac{\pi}{M}$. In Rayleigh fading channel the signal envelop is Rayleigh distributed and the instantaneous SNR is Chi-square (John Proakis 1983), so the probability density function of SNR, $\gamma$ has the form

$$
p(\gamma)=\frac{1}{\rho} \exp \left(-\frac{\gamma}{\rho}\right) \quad \gamma \geq 0
$$

Where $\rho$ is the average SNR. The average conditional error rate over the random variable $\gamma$ is

$$
P_{e}=\int_{0}^{\infty} P_{e}(\gamma) p(\gamma) d \gamma
$$

To obtain the error rate using Eq. (13), a double integral must be evaluated. Interchanging the order of integration with respect to the variable $\gamma$ can be carried out first

$$
\text { PSK: } P_{e}=\frac{1}{\pi} \int_{-\frac{\pi}{2}}^{\frac{\pi}{2}-\alpha} \frac{\cos ^{2} \theta}{\cos ^{2} \theta+\rho \sin ^{2} \alpha} d \theta
$$

The remaining single integral can evaluate with the aid of integration table [15] yielding PSK:

$P_{e}=\frac{M-1}{M}-\frac{1}{\pi} \sqrt{\left(\frac{\rho \sin ^{2} \alpha}{1+\rho \sin ^{2} \alpha}\right)} \times\left\{\frac{\pi}{2}+\sin ^{-1}\left[\sqrt{\left(\frac{\rho}{1+\rho}\right)} \cos \alpha\right]\right\}$

For binary modulation, the Eq. (15) can be reduce to the following result (John Proakis 1983)

$$
P_{e}=\frac{1}{2}\left[1-\sqrt{\left(\frac{\rho}{1+\rho}\right)}\right]
$$

After simplification and substituting $=\frac{\pi}{M}$, the error rates at high SNR $(\rho>10 \mathrm{~dB})$ assume the form PSK:

$$
P_{e} \approx\left(\frac{M-1}{M}\right)\left\{1-\sqrt{\left[\frac{\rho \sin ^{2}\left(\frac{\pi}{M}\right)}{1+\rho \sin ^{2}\left(\frac{\pi}{M}\right)}\right]}\right\}
$$

\section{16-PSK (Phase Shift Keying) Modulation Schemes}

It is a one type of $M$-ary PSK where $M=16$. In 16-PSK modulation scheme we can send $\left(k=\log _{2} M=\log _{2} 16=4\right) 4$ bit 
information per symbol. In Fig. 4 and Fig. 5, symbol constellation and gray coded bit mapping for 16-PSK is shown respectively.

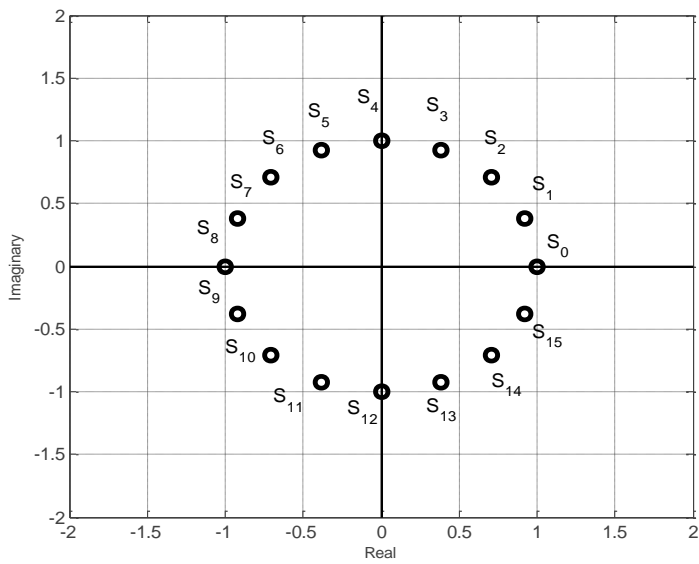

Figure 2: 16-PSK constellation plot

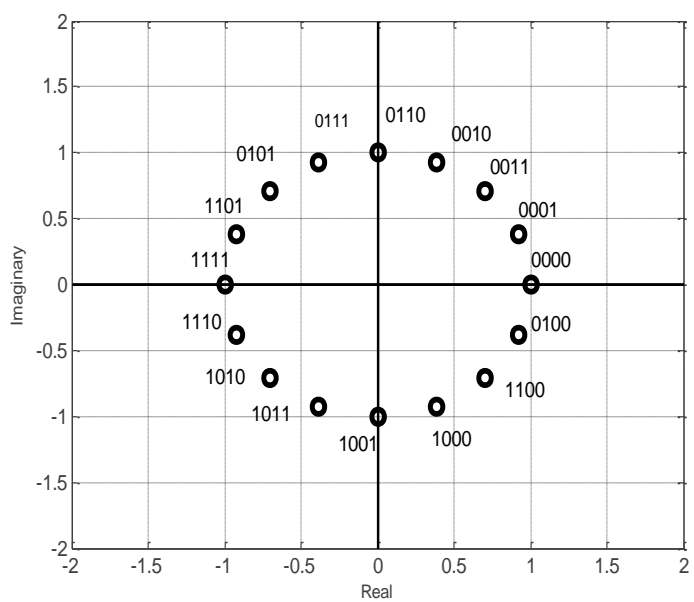

Figure 3: Gray coded bit mapping for 16-PSK

\section{F. M-ary QAM Modulation in OFDM Model}

The general analytic expression for M-ary QAM waveform is: $s_{i}(t)=A_{m c} \cos \omega_{c} t-A_{m s} \sin \omega_{c} t, 0 \leq 0 t \leq 0 T_{s}$ 18)

,where $i=0,1,2, \ldots, M$ and $T_{S}$ is the symbol interval, which is related to the bit interval by $T_{s}=m T_{b}$, where $m=$ $\log _{2} M$ represents the number of bits per symbol. The quadrature amplitudes $A_{m c}$ and $A_{m s}$ range over a set of $\{ \pm d, \pm 3 d, \pm 5 d, \ldots$.$\} , in which 2 d$, represents the minimum Euclidean distance of constellation points, and $d$ can be calculated as: (H. G. Yeh and H. S. Seo) $d=\sqrt{\frac{3 m E_{b}}{2(M-1)}}$ $E_{b}$ : average energy bit. As a result, the 16-QAM bandwidth efficiency is 4 bits/ $\mathrm{Hz}$ and 64-QAM bandwidth efficiency is 6 bits/Hz.

\section{SER calculation for M-ary QAM in Rayleigh fading channel}

Communication system used in this paper employs M-ary signaling over a Rayleigh fading channel. Besides the fading, the signal is also perturbed by AWGN with two sided Power Spectral Density (PSD) $\frac{N_{0}}{2}$. For a digital receiver (J. Sun, et al) which makes its decision based on the output of a linear filter operating on an undistorted symbol waveform, it is well known that the probability of symbol error caused by stationary AWGN depends only on the instantaneous SNR $\gamma_{s}$ associated with each symbol. In fading, $\gamma_{s}$ become a random variable and its PDF for Rician channel is [18],

$p\left(\gamma_{s}\right)=\left(\frac{1+K}{\overline{\gamma_{s}}}\right) \exp \left[-\frac{(1+K) \gamma_{s}+K \overline{\gamma_{s}}}{\overline{\gamma_{s}}}\right] \cdot I_{0}\left(2 \sqrt{\frac{K(1+K) \gamma_{s}}{\overline{\gamma_{s}}}}\right)$

Once the PDF of $\gamma_{s}$ is known, the average SER in fading can be calculated by averaging the conditional (on $\gamma_{s}$ ) SER over the $\operatorname{PDF} \gamma_{s}$ i.e.

$$
P_{s}(e)=\int_{0}^{\infty} P_{s}\left(\frac{e}{\gamma_{s}}\right) p\left(\gamma_{s}\right) d \gamma_{s}
$$

Where $P_{S}\left(\frac{e}{\gamma_{s}}\right)$ is the conditional (on $\gamma_{s}$ ) SER in non fading channel corrupted by AWGN. Using an alternate representation for the square of the Gaussian Q-function (M. K. Simon and D. Divsalar 1998), the conditional SER for the fading channel is given by (M. K. Simon and M. S. Alouini 1999),

$P_{S}\left(\frac{e}{\gamma_{s}}\right)=\frac{4 B}{\pi} \cdot \int_{0}^{\frac{\pi}{2}} \exp \left(-\frac{\mathrm{g} \gamma_{S}}{\sin ^{2} \theta}\right) d \theta-\frac{4 B^{2}}{\pi} \cdot \int_{0}^{\frac{\pi}{4}} \exp \left(-\frac{\mathrm{g} \gamma_{S}}{\sin ^{2} \theta}\right) d \theta$

where $\mathrm{g}=3 /[2(M-1)]$ and $B=1-1 / \sqrt{M}$. Now the substitution of (19) and (20) into (21) and considering $K=0$ yields the average SER of M-QAM over a Rayleigh fading channel, which is given as,

$\mathrm{P}_{\mathrm{S}}(\mathrm{e})=\frac{4 B}{\pi} \cdot \int_{0}^{\frac{\pi}{2}} \frac{\sin ^{2} \theta}{\sin ^{2} \theta+\mathrm{g} \overline{\gamma_{s}}} \mathrm{~d} \theta-\frac{4 B^{2}}{\pi} \cdot \int_{0}^{\frac{\pi}{4}} \frac{\sin ^{2} \theta}{\sin ^{2} \theta+\mathrm{g} \overline{\gamma_{s}}} \mathrm{~d} \theta$

\section{16-QAM (Quadrature Amplitude Modulation) Modulation scheme}

It is a one type of M-ary QAM, where $M=16$. In 16-QAM modulation scheme we can send $\left(k=\log _{2} M=\log _{2} 16=4\right) 4$ bit information per symbol. In Fig. 4 and Fig. 5, symbol constellation and gray coded bit mapping for 16-QAM is shown respectively. 


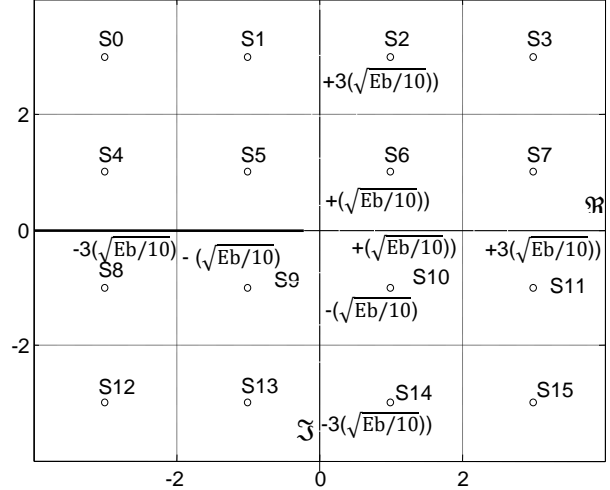

Figure 4: 16-QAM constellation

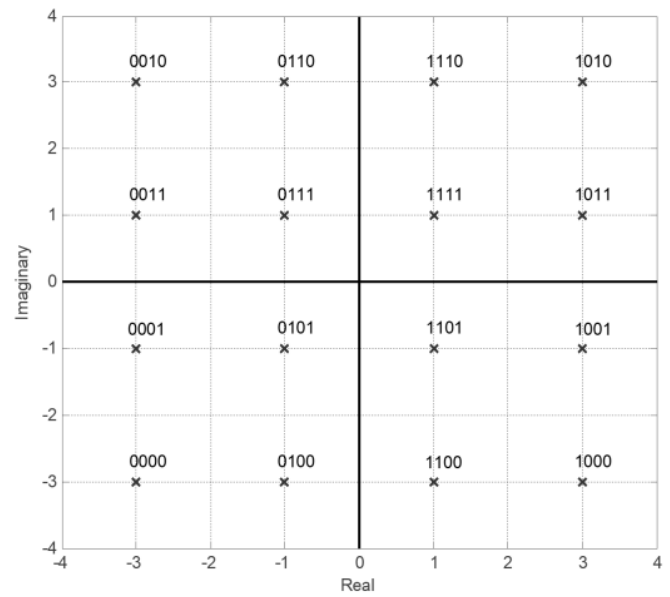

Figure 5: Gray coded bit mapping for 16-QAM

\section{RESULT AND SIMULATION}

To analyze the performance of different modulation schemes with gray coded bit mapping and without using gray coded mapping with OFDM technique in Rayleigh fading channel, we perform simulations using MATLAB.

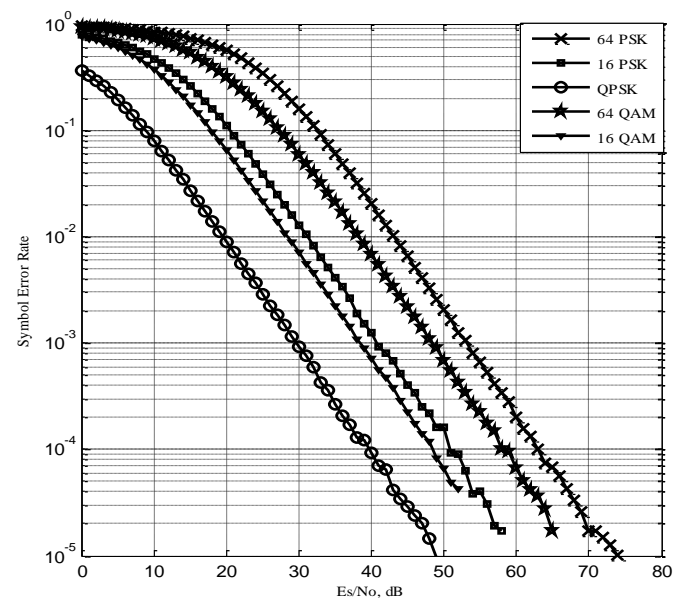

Figure 6: SER curve for different modulation scheme using OFDM technique
From Fig.6 we can see that after performing MATLAB simulation for 64 PSK the signal to noise ratio is $64 \mathrm{~dB}$ and for 64 QAM it is $58 \mathrm{~dB}$. Again for 16 PSK the signal to noise ratio is $52 \mathrm{~dB}$ and for 16 QAM it is $48 \mathrm{~dB}$.

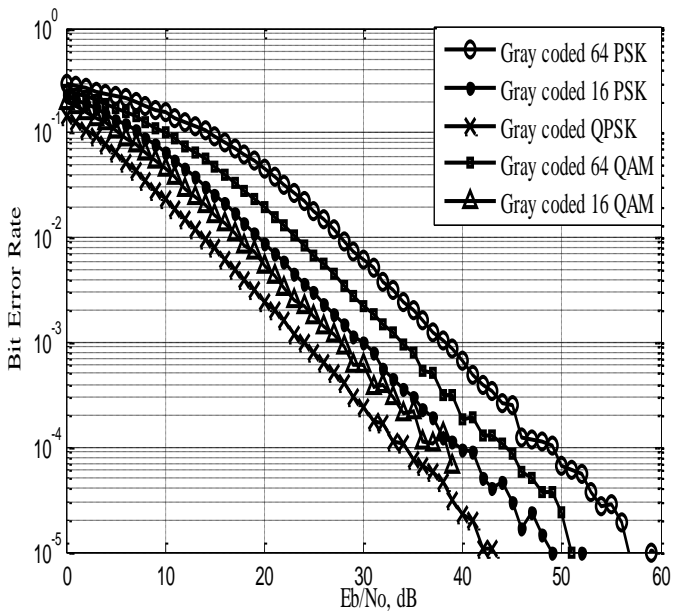

Figure 7: BER curve for different modulation Scheme using OFDM with gray coded bit mapping.

From Fig.7 we can see that after performing MATLAB simulation with gray bit mapping for 64 PSK the signal to noise ratio is $48 \mathrm{~dB}$ and for 64 QAM it is $45 \mathrm{~dB}$. Again for 16 PSK the signal to noise ratio is $38 \mathrm{~dB}$ and for $16 \mathrm{QAM}$ it is $35 \mathrm{~dB}$. The gain from SER and BER in different modulation scheme is summarized in Table-II

Table-II: SER and BER for different modulation scheme

\begin{tabular}{c|c|c|c}
\hline $\begin{array}{c}\text { Different modulation } \\
\text { scheme }\end{array}$ & $\begin{array}{c}\text { SER } \\
\text { at } 10^{-4}\end{array}$ & $\begin{array}{c}\text { BER } \\
\text { at } 10^{-4}\end{array}$ & $\begin{array}{c}\text { Gain from } \\
\text { SER \& } \\
\text { BER }\end{array}$ \\
\hline 64-PSK & $64 \mathrm{~dB}$ & $49 \mathrm{~dB}$ & $13 \mathrm{~dB}$ \\
64-QAM & $58 \mathrm{~dB}$ & $45 \mathrm{~dB}$ & $12 \mathrm{~dB}$ \\
16-PSK & $52 \mathrm{~dB}$ & $40 \mathrm{~dB}$ & $12 \mathrm{~dB}$ \\
16-QAM & $48 \mathrm{~dB}$ & $37 \mathrm{~dB}$ & $11 \mathrm{~dB}$ \\
QPSK & $40 \mathrm{~dB}$ & $34 \mathrm{~dB}$ & $6 \mathrm{~dB}$ \\
\hline
\end{tabular}

\section{CONCLUSION}

Here we have analyzed the performance of different modulation schemes with OFDM technique in Rayleigh fading channel with gray coded bit mapping and without gray mapping. By using higher order modulation scheme (like 16-QAM, 64-QAM, 16PSK, 64-PSK) with OFDM technique in Rayleigh channel, we can transmit more data rate. In both the cases we get better performance for QAM modulation scheme. So, at this stage we can easily conclude that QAM has got better performance than PSK and use of gray bit mapping enhances this performance even more. 


\section{REFERENCES}

R. D. Van Nee and R.Pras a d "OFDMfor Winless Multimedia Communications”. Artech House, Januaruy 2000.

John A.C Bingham, "Multicarrier modulation for data transmission: An idea whose time has come ." IEEE Communication Magazine, May 1990,vol.28, issue 5, pp 5-14.

Mehul Jain and M.Mani Roja, "Comparison of OFDM with CDMA system in Wireless Telecommunication for multipath delay spread, The first IEEE and IFIP International Conference in Central Asia, 26-29 Sept 2005, pages5

Z.Wang and G. B. Giannakis, "Wireless multicarrier communications," IEEE Signal Processing Mag., pp. 29-48, May 2000.

A. Ruiz, J. M. Cioffi, and S. Kasturia, "Discrete multiple tone modulation with coset coding for the spectrally shaped channel," IEEE Trans. Commun., vol. 40, pp. 1012-1029, June 1992.

Dwight, H.B.:" Tables of integrals and other mathematical data", Macmillan Co.,N.Y.,1961, pp. 105-106.

ETSI, Broadband radio access network (BRAN): HYPERLAN Type 2 functional specification Part 1-Physical layer, DTS/BRAN 030 003-1, June 1999.

ETSI, Digital video broadcasting (DVB-T); Framing structure, channel coding, and modulation for digital terrestrial television, ETS 300 744, Dec. 2001.

ETSI, Radio broadcast systems; Digital audio broadcasting (DAB) to mobile, portable, and fixed receivers, ETS 300 401, May 1997.

H. G. Yeh and H. S. Seo, "Low Complexity Demodulator for M-ary QAM", Proc. of Wireless Telecommunication Symposium, April. 2007,Pomona, CA.
J. Sun, et al," Performance of MDPSK, MPSK, non-coherent MFSK in wireless Rician fading channels," IEEE Trans. Commun. vol. 47, pp 813-816, June 1999.

John Proakis, "Digital Communications", McGraw-Hill, N.Y., 1983, pp. 466-468.

John-R-Barry, Edward A. Lee, David G. Messerschmitt, Digital Communication, $3^{\text {rd }}$ Edition.

L. J. Cimini Jr., "Analysis and simulation of a digital mobile channel using orthogonal frequency division multiplexing," IEEE Trans. Commun., vol. 33, pp. 665-675, July 1985.

M. K. Simon and D. Divsalar, "Some new twists to problems involving the Gaussian probability integral," IEEE Trans. Commun. vol. 46, pp 200-210, Feb. 1998.

M. K. Simon and M. S. Alouini, "A unified performance analysis of digital communication with dual selective combining diversity over correlated Rayleigh and Nakagami m fading channels," IEEE Trans. Commun.vol. 47, pp 33 43, Jan. 1999.

Nor K. Noordin, Borhanuddin M. Ali, S. S. Jamuar, Tharek.A.Rahman \& Mahamod B. Ismail," Preliminary Investigation Of Gray Coded M-QuadratureAmplitude Modulation In Orthogonal Frequency Division Multiplexing Over Additive White Gaussian Noise Channel" Jurnal Teknologi, 40(D) Jun. 2004: 49-58 .

Pawula, R.F., Rice,. O., and Roberts, J. H.:" Distribution of the phase angle between two vectors perturbed by Gaussian noise", IEEE Trans.,1982, Com-30, pp. 1828-1841.

Weinstein, S.B., Ebert, P.M., Data Transmission by FrequencyDivision Multiplexing Using the Discrete Fourier Transform, IEEE Transactions on Communication Technology, Vol. COM-19, no. 5, pp. 628-634, October 1971. 